\title{
EVALUACIÓN DE DIFERENTES NIVELES CRÍTICOS DE Empoasca spp. BASADO EN PORCENTAJE DE HOJAS INFESTADAS CON NINFAS EN EL CULTIVO DE FRIJOL
}

\author{
R. Escobar2, O. Cáceres, K. Andrews y R. Cave
}

\section{COMPENDIO}

En la época de primera de 1988 se realizaron ensayos en frijol para determinar el nivel crítico óptimo para el control de Empoasca spp., basado en el muestreo del porcentaje de hojas infestadas con ninfas. También se evaluó la eficacia de este muestreo comparándolo con un muestreo absoluto usando una trampa de cuña. Se utilizó un diseño experimentai de bloques completos al azar con dos localidades, tres repeticiones en cada lugar y cinco tratamientos; aplicaciones de insecticida a $0,15,30$ y $45 \%$ de hojas Infestadas y un testigo sin aplicación. A cada tratamiento se le aplicó dimetoato al alcanzar su nivel crítico. El tamaño de cada parcela fue de $49 \mathrm{~m}^{2}$ y para la cosecha se utilizó una área útil de $4 \mathrm{~m} 2$ del centro de cada parcela. El análisis combinado de varianza indica que hubo diferencias significativas $(\mathrm{P} \leq 0.01)$ en rendimiento entre los tratamientos. Los rendimientos de 0 y $5 \%$ (1.1 y $1.2 \mathrm{t} / \mathrm{ha}$, respectivamente) fueron significativamente más altos que el testigo ( $0.8 \mathrm{tm} / \mathrm{ha})$, no así para los tratamientos $30 \%$ y $45 \%$. La correlación entre el porcentaje de hojas infestadas por ninfas y el número de ninfas determinado por el muestreo absoluto fue significativa $(r=0.78)$ en Corral Falso y San Jerónimo $(r=036)$. El tratamiento con $15 \%$ de hojas infestadas obtuvo los mayores beneficios netos. La tasa de retorno marginal para este nivel crítico fue 15 y $20 \%$ para San Jerónimo y Corral Falso, respectivamente.

Palabras clave adicionales: Muestreo, Phaseolus vulgaris.

\section{INTRODUCCIÓN}

El Salta hojas (Empoasca spp.) es considerada la plaga más importante del frijol en América Tropical. Se han reportado pérdidas en el rendimiento de $57 \%$ sin utilizar ninguna protección química (Schoonhoven y Cardona, 1985).

El método de control más utilizado es el uso de insecticidas, pero no existe un método práctico y sencillo para determinar los niveles críticos y así decidir cuando aplicar. King y Saunders (1984) reportaron que se deben hacer aplicaciones cuando hay un promedio de una o más ninfas por hoja trifoliada en cada 20 hojas contadas en el campo.

Andrews (1984) recomendó revisar semanalmente 100 hojas trifoliadas, o 100 plantas distribuidas en diez lugares para cuantificar ninfas o adultos, respectivamente. Con este método de muestreo se recomiendan niveles críticos promedios de un adulto/planta hasta la aparición de las primeras hojas verdaderas, 2 adultos/planta durante el período vegetativo y la floración y 3 adultos/planta durante el llenado de las vainas. En el ca-

\begin{abstract}
In May, 1988, trials were Initiated to determine optimum critical levels for control of Empoasca spp, based on percentage of nymph Infested leaves. The efficiency of this sampling comparing It to absolute values obtained from Insect traps was also evaluated. A randomized complete block design was employed at two locations with three replications and five treatments per location. Insecticides were applied when leaves had infestation percentages of $0,15,30$ and $45 \%$. An added check was also included without insecticide applications. To each treatment, "dimetoato" was applied to determine the critical level. Plot sizes were 49 in' while harvest areas were $4 \mathrm{~m} 2$ taken from the center of each plot. The combined analysis of variance Indicated significant differences between yield amongst the applied treatments $(\mathrm{P}<0.01)$. Yield increases of 0 and $5 \%$ ( 1.1 and $1.2 \mathrm{t} \mathrm{ha}^{-1}$, respectively) were significantly higher than the check $(0.8$ $\mathrm{t} / \mathrm{ha}$ ) for treatments 30 and $45 \%$ defined above. The correlation between porcentage of infested leaves and number of nymphs determined from the traps was significant $(r=0.78)$ at Corral Falso and $(r=0.56)$ at San Jeronimo. The treatment with $15 \%$ infested leaves provided the largest net benefits. The marginal rate of return for this critical level was 5 and $20 \%$ for San Jeronimo and Corral Falso respectively.
\end{abstract}

so de las ninfas se recomienda 2 ninfas/hojas trifoliada desde la aparición de las dos hojas verdaderas hasta la producción de las primeras vainas y 3 ninfas/hoja trifoliada durante el llenado de las vainas, Andrews y Barletta (1985) recomendaron a campesinos la metodología descrita anteriormente, pero unificando los niveles críticos, quedando 3 ninfas/hoja trifoliada o 3 adultos por planta, sin importar la etapa fenológica del cultivo.

Esta recomendación fue difícil de transmitir a pequeños agricultores debido a que es necesario hacer cálculos, además que los adultos vuelan rápidamente y es difícil contarlos; también hay confusión con otros cicadélidos e insectos pequeños. Debido a estas dificultades en la transferencia de tecnología el personal de la EAP inició estudios para determinar la relación entre el porcentaje de hojas infestadas por ninfas y el número de ninfas/hoja trifoliada. Sobrado et al (1986) reportó un

1 Presentado en la XXXV Reunión Anual M PCCMCA, San Pedro Sula, Honduras. 1989

2 Departamento de Protección Vegetal, Escuela Agrícola Panamericana, El Zamorano. Apanado 93, Tegucigalpa, Honduras.

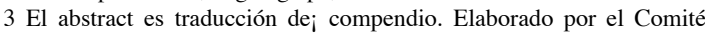
Editorial para mostrar el formato de presentación de los artículos.

Publicado en Agronomía Mesoamericana, Vol. 1 (1990). 
método de muestreo absoluto de adultos de Empoasca spp. que permite a la vez hacer muestreo de ninfas y otras plagas en el follaje y el suelo. Este muestreador tipo cuña se coloca sobre las plantas de frijol y se sacuden manualmente las plantas por la ventana posterior, los adultos de salta hojas que son positivamente fototácticos vuela hacia la cubierta de plástico donde son fácilmente contados. Portillo (1988), encontró una relación lineal y positiva entre la población de ninfas/hoja trifoliada y el porcentaje de hojas trifoliadas infestadas. Sin embargo, con la mayor población de ninfas/hoja trifoliada que se logró medir (2.4) el porcentaje de hojas infestadas no llegó a $100 \%$.

Basado en lo anterior, Portillo (1988) hizo un estudio para establecer un método de muestreo de utilidad práctica para los agricultores y que a la vez mida las poblaciones de Empoasca spp. obtenidos con los métodos convencionales. El sugiere que el nivel crítico a ser usado con este método es de $30 \%$ de las hojas trifoliadas totales infestadas con ninfas, esto es equivalente al nivel crítico convencional de tres ninfas/ hoja trifoliada recomendado por Andrews y Barletta (1985).

El presente estudio fue un seguimiento del método de muestreo planteado por Portillo (1988). Los objetivos fueron: 1) Determinar la disminución en rendimiento de frijol causada por el ataque de diferentes niveles poblacionales de Empoasca spp., 2) Comparar un método basado en el porcentaje de hojas trifoliadas con ninfas con el método de muestreo absoluto y 3 ) Determinar el nivel crítico óptimo para el control de Empoasca spp.

\section{MATERIALES Y MÉTODOS}

\section{Descripción del área experimental}

El experimento se llevó a cabo en seis sitios, tres no incluidos por falta desaltahojas y uno se perdió por problemas con el agricultor. Los experimentos restantes se sembraron el 18 y 27 de junio de 1988 en las aldeas de Corral Falso y San Jerónimo, respectivamente, jurisdicción de San Matías, departamento de El Paraíso, Honduras.

\section{Diseño experimental}

En cada sitio se utilizó un diseño experimental de bloques completamente al azar con dos réplicas y tres repeticiones. El tamaño de cada parcela fue de $49 \mathrm{~m} 2$ y el área de cosecha de $4 \mathrm{~m}_{2}$. El experimento se condujo a nivel de finca, la siembra, las prácticas agronómicas y culturales realizadas en el ensayo, excepto las aplicaciones de insecticidas, fueron hechas por el agricultor dueño del lote. Se usaron cinco tratamientos: Aplicaciones de dimetoato con dosis de $11 /$ ha al alcanzar el nivel de $0,15,30$ y $45 \%$ de hojas infestadas y un testigo sin aplicación
El porcentaje de infestación se determinó observando el envés de 50 hojas trifoliadas en cinco sitios dentro de cada parcela, al mismo tiempo se realizó el muestreo absoluto en cuatro sitios dentro de cada parcela donde se determinó el número de ninfas por hoja trifoliada y adultos por planta. Ambos muestreos (absoluto y relativo) se comenzaron a hacer desde la etapa fenológica V4 dos veces a la semana.

\section{RESULTADOS Y DISCUSIÓN}

Utilizando el método de muestreo relativo para hacer las aplicaciones al alcanzar el nivel crítico, el análisis combinado de varianza en las dos localidades nos indica que hubo diferencias significativas $(\mathrm{P} \leq 0.01)$ para el rendimiento, entre los tratamientos. El tratamiento con $15 \%$ de hojas infestadas resultó en los mayores rendimientos $(1.2 \mathrm{tm} / \mathrm{ha})$ y junto al tratamiento $0 \%(1.1$ $\mathrm{tm} / \mathrm{ha}$ ) fueron significativamente más altos que el testigo sin aplicación $(0.8 \mathrm{tm} / \mathrm{ha})$ (Cuadro 1$)$, También el tratamiento $15 \%$ fue diferente estadísticamente que los tratamientos con niveles críticos más altos. La diferencia en rendimiento entre el tratamiento que obtuvo el más alto y el más bajo fue de $0.4 \mathrm{tm} / \mathrm{ha}$ que equivale a una reducción de $33 \%$.

Cuadro 1. Rendimientos de frijol común (14\% de humedad) promedios en lotes donde se usaron cinco niveles críticos para aplicación contra Empoasca spp. Departamento de El Paraíso, Honduras, C.A. 1988

\begin{tabular}{cc}
\hline $\begin{array}{c}\text { Tratamientos } \\
\text { (Porcentaje de hojas } \\
\text { infestadas con ninfas) }\end{array}$ & $\begin{array}{c}\text { Rendimientos } \\
\text { (tm/ha) }\end{array}$ \\
\hline $15 \%$ & $1.2 \mathrm{a}$ \\
$0 \%$ & $1.1 \mathrm{a} \mathrm{b}$ \\
$30 \%$ & $0.9 \quad \mathrm{~b} \mathrm{c}$ \\
$45 \%$ & $0.9 \quad \mathrm{c}$ \\
Sin aplicación & $0.8 \quad \mathrm{c}$ \\
\hline
\end{tabular}

$\mathrm{a}=$ Rendimientos con letras iguales son estadísticamente iguales.

La correlación entre el número de ninfas por hoja trifoliada determinado por el muestreo absoluto y el porcentaje de hojas infestadas determinado por el muestreo relativo fue de $r=0.78$ y $r=0.56$ para Corral Falso y San Jerónimo, respectivamente, que son similares a las encontradas por Portillo(1988). Asimismo, se hicieron correlaciones entre el número de ninfas por hoja trifoliada determinado por el muestreo absoluto y el número de adultos determinado por el mismo muestreo. Los coeficientes de correlación fueron bajos $(r=$ 0.2 y $r=0.3$ para Corral Falso y San Jerónimo, respectivamente), que no coinciden con lo encontrado por Portillo (1988), probablemente porque las poblaciones de adultos no fueron suficientemente altas durante todas las fases de desarrollo del cultivo, cabe mencionar 
que Portillo (1988) realizó su experimento en la época de verano, donde las poblaciones son extremadamente altas, porque no hay efecto de la lluvia y tampoco hay cultivos de frijol alrededor.

Se hizo un análisis económico para determinar con que tratamiento se obtuvieron los mayores beneficios netos (Cuadro 2). El presupuesto parcial para cada uno de los tratamientos indica que en ambas localidades, el tratamiento con aplicaciones al 15\% de hojas infestadas obtuvo los mayores beneficios netos con US\$ 797 y US\$ 1055 para San Jerónimo y Corral Falso, respectivamente.

En el Cuadro 3 se muestra un análisis marginal para cada tratamiento en las dos localidades, el tratamiento con aplicaciones al $15 \%$ de hojas infestadas obtuvo la tasa de retorno marginal más alta en ambas localidades. Por US $\$ 1.00$ invertido se recibió US\$ 15.00 y US\$ 20.00 de ganancia en San Jerónimo y Corral Falso respectivamente.

En promedio, para las dos localidades, el tratamiento con aplicaciones al 15\% de hojas infestadas, siempre obtuvo los mayores beneficios netos y la tasa de retorno marginal más alta (Cuadros 4 y 5, respectivamente). Estos resultados no coinciden con los resultados obtenidos por Portillo (1988), en los cuales se obtuvo como nivel crítico el $30 \%$ de hojas infestadas. Esta diferencia se debió a que se hizo 3.15 aplicaciones en promedio a las parcelas con nivel crítico de $15 \%$ de hojas infestadas, mientras que a las parcelas con $30 \%$ se les hizo 2.7 aplicaciones en promedio (Cuadro 4).

Cuadro 2. Presupuesto parcial para cinco niveles críticos de aplicación para Empoasca spp. en dos localidades. Se basó en cálculos deprecio de venta de U. $\$ 4 / 0.82 \mathrm{~kg}$

\begin{tabular}{|c|c|c|c|c|c|c|c|c|c|c|}
\hline \multirow[b]{2}{*}{ Concepto } & \multicolumn{2}{|c|}{$0 \%$} & \multicolumn{2}{|c|}{$15 \%$} & \multicolumn{2}{|c|}{$30 \%$} & \multicolumn{2}{|c|}{$45 \%$} & \multicolumn{2}{|c|}{ Sin aplicación } \\
\hline & S.J.a & C.F.b & S.J. & C.F. & S.J. & C.F. & S.J. & C.F. & S.J. & C.F. \\
\hline \multicolumn{11}{|l|}{ Beneficios } \\
\hline Rendimiento $\mathrm{kg} / \mathrm{ha}$ & 1058.10 & 1106.00 & 1039.80 & 1361.10 & 749.50 & 1078.00 & 927.90 & 966.90 & 703.70 & 809.40 \\
\hline Valor \$ & 0.82 & 0.82 & 0.82 & 0.82 & 0.82 & 0.82 & 0.82 & 0.82 & 0.82 & 0.82 \\
\hline Beneficio total \$ & 867.60 & 906.90 & 852.60 & 1116.10 & 614.60 & 883.90 & 760.50 & 792.80 & 577.00 & 663.70 \\
\hline \multicolumn{11}{|l|}{ Costos variables } \\
\hline Cantidad (1 1/ha) & 9.00 & 8.00 & 3.00 & 3.30 & 2.70 & 2.70 & 2.70 & 1.70 & 0.00 & 0.00 \\
\hline Valor \$ & 15.00 & 15.00 & 15.00 & 15.00 & 15.00 & 15.00 & 15.00 & 15.00 & 0.00 & 0.00 \\
\hline Total \$ & 135.00 & 120.00 & 45.00 & 49.50 & 40.50 & 40.50 & 40.50 & 25.50 & 0.00 & 0.00 \\
\hline \multicolumn{11}{|l|}{ Mano de obra } \\
\hline Cantidad de días & 12.60 & 11.20 & 4.20 & 4.60 & 3.70 & 3.80 & 3.70 & 2.40 & 0.00 & 0.00 \\
\hline Valor \$ & 2.50 & 2.50 & 2.50 & 2.50 & 2.50 & 2.50 & 2.50 & 2.50 & 0.00 & 0.00 \\
\hline Total \$ & 31.50 & 28.00 & 10.50 & 11.50 & 9.25 & 9.50 & 9.25 & $6 \mathrm{M}$ & 0.00 & 0.00 \\
\hline Total costos variables & 166.50 & 148.00 & 55.50 & 61.00 & 49.75 & 50.00 & 49.75 & 31.50 & 0.00 & 0.00 \\
\hline Beneficio neto & 701.10 & 758.90 & 797.10 & 1055.10 & 564.80 & 883.90 & 751,25 & 761.30 & 557.00 & 663.70 \\
\hline
\end{tabular}

$\mathrm{a}=$ Localidad de San Jerónimo b = Localidad de Corral Falso.

Cuadro 3. Análisis marginal para cinco niveles críticos de aplicación contra Empoasca spp. en el departamento de El Paraíso, Honduras, C.A. 1988

\begin{tabular}{lcrrrrr}
\hline Localidad Trata- Benefi- & $\begin{array}{c}\text { Costos } \\
\text { miento cio neto caria- } \\
\text { bles }\end{array}$ & $\begin{array}{c}\text { Incremento } \\
\text { marginal } \\
\text { en bene- } \\
\text { ficio neto }\end{array}$ & $\begin{array}{c}\text { Incremento } \\
\text { marginal } \\
\text { en costos } \\
\text { variables } \\
\text { Marginal }\end{array}$ & $\begin{array}{c}\text { Tasa } \\
\text { Re- } \\
\text { torno }\end{array}$ \\
& \multicolumn{7}{c}{} & & & & & \\
& & & & & & \\
& & & & & & \\
San & $15 \%$ & 797.10 & 55.50 & 45.80 & 5.75 & 15.10 \\
Jeró_ & $45 \%$ & 710.75 & 49.75 & 153.75 & 49.75 & 3.00 \\
nimo & $0 \%$ & 701.10 & $165.50 \mathrm{a}$ & & & \\
& $30 \%$ & 564.80 & $49.75 \mathrm{a}$ & & & \\
& Sin Aplic. & 557.00 & 0.00 & & & \\
Corral & $15 \%$ & 1055.10 & 61.00 & 221.20 & 11.00 & 20.10 \\
Falso & $30 \%$ & 833.90 & 50.00 & 72.60 & 18.50 & 3.90 \\
& $45 \%$ & 761.30 & 31.50 & 98.30 & 31.50 & 3.10 \\
& $0 \%$ & 758.90 & $148.00 \mathrm{a}$ & & \\
& $\mathrm{T}$ & 663.00 & 0.00 & & & \\
\hline
\end{tabular}

$\mathrm{a}=$ No se tornan en cuenta por dominancia.
Cuadro 4. Presupuesto parcial para cinco niveles críticos de aplicación para Empoasca spp. en promedio para dos localidades. Se basó en cálculos de precio de venta de \$0.50/kg en El Paraíso, Honduras, C.A. 1988

\begin{tabular}{lrrrrr}
\hline \multicolumn{1}{c}{$\begin{array}{c}\text { Concepto } \\
\text { Beneficios }\end{array}$} & \multicolumn{1}{c}{ 0\% } & $\mathbf{1 5 \%}$ & $\mathbf{3 0 \%}$ & $\mathbf{4 5 \%}$ & $\begin{array}{c}\text { Sin } \\
\text { Aplicación }\end{array}$ \\
\hline Rendimiento kg/ha & 1082.07 & 1200.48 & 913.78 & 947.43 & 756.58 \\
Valor \$ & 0.82 & 0.82 & 0.82 & 0.82 & 0.82 \\
Beneficio Total \$ & 887.30 & 984.39 & 749.30 & 776.89 & 620.39 \\
Costos Variables & & & & & \\
Insecticida & & & & & \\
Cantidad (1 1/ha) & 8.50 & 3.15 & 2.70 & 2.20 & 0.00 \\
Valor \$ & 15.00 & 15.00 & 15.00 & 15.00 & 0.00 \\
Total \$ & 127.50 & 47.25 & 40.50 & 33.00 & 0.00 \\
Mano de Obra & & & & & \\
Cantidad de días & 11.90 & 4.40 & 3.75 & 3.05 & 0.00 \\
Valor \$ & 2.50 & 2.50 & 2.50 & 2.50 & 0.00 \\
Total \$ & 29.75 & 11.00 & 9.37 & 7.62 & 0.00 \\
Total costos variables 157.25 & 58.25 & 49.62 & 40.62 & 0.00 \\
Beneficio Neto & 857.55 & 926.14 & 669.68 & 736.27 & 620.39 \\
\hline a = Mismo número de Aplicaciones & & & \\
\hline
\end{tabular}


Cuadro 5. Análisis marginal para cinco niveles críticos de aplicación para Empoasca spp. en promedio para dos localidades. El Paraíso Honduras, C.A. 1988

\begin{tabular}{cccccc}
\hline Tratamiento & $\begin{array}{c}\text { Benefi- Costos } \\
\text { cio } \\
\text { Neto }\end{array}$ & $\begin{array}{c}\text { varia- } \\
\text { bles }\end{array}$ & $\begin{array}{c}\text { Incremento } \\
\text { marginal } \\
\text { en bene- } \\
\text { ficio neto }\end{array}$ & $\begin{array}{c}\text { Incremento } \\
\text { marginal } \\
\text { en vostos } \\
\text { variables }\end{array}$ & $\begin{array}{c}\text { Tasa de } \\
\text { retorno } \\
\text { marginal }\end{array}$ \\
\hline & & & & & \\
\hline 15 & 926.14 & 58.25 & 189.87 & 17.63 & 10.70 \\
0 & 857.55 & 157.25 a & & & \\
45 & 736.27 & 40.62 & 115.88 & 40.62 & 2.80 \\
30 & 669.68 & $49.62 \mathrm{a}$ & & & \\
Sin aplicac. & 620.39 & 0.00 & & & \\
\hline
\end{tabular}

$\mathrm{a}=$ No se toman en cuenta dominancia

\section{CONCLUSIONES Y RECOMENDACIONES}

1. Empoasca spp. redujo el rendimiento del cultivo de frijol en $0.44 \mathrm{tm} / \mathrm{ha}$, o sea un 33\%, que es una pérdida muy cerca a la reportada por otros autores. Esta pérdida se le atribuye a Empoasca spp. porque la presencia de otras plagas fue insignificante en las parcelas sin aplicación.

2. El tratamiento con aplicaciones al $15 \%$ de hojas infestadas obtuvo los mayores rendimientos, el mayor beneficio neto y la más alta tasa de retorno marginal. Sin embargo, no se puede recomendar este nivel crítico de aplicación, ya que en la época de postrera de 1988 se llevaron a cabo 3 experimentos más, pero no se presentó la plaga, por lo que se recomienda seguir este estudio durante la siguiente temporada, sobre todo en la época de postrera, que es cuando más se siembra el cultivo del frijol.

3. Se encontró alta correlación positiva entre el número de ninfas por hoja trifoliada, determinado por el muestreo absoluto y el porcentaje de hojas infestadas determinado por el muestreo relativo que coinciden por lo encontrado por Portillo (1988). Sin embargo, la correlación entre las poblaciones absolutas de adultos/planta y ninfas/hoja trifoliada fueron bajas, y no coinciden con lo encontrado por Portillo (1988). Esto es muy importante, porque Portillo (1988) para obtener el 30\% de hojas infestadas corno nivel crítico de aplicación, consideró una alta co- rrelación entre el número de adultos/planta y el número de ninfas/hoja trifoliada. Sin embargo, estas diferencias se pueden atribuir a que Portillo hizo su estudio en la época de verano, donde las poblaciones son más estables, no hay interferencia de lluvia y no hay más cultivos alrededor.

Asimismo, Portillo (1988) reporta que el método puede dar recomendaciones diferentes en cuanto a la decisión de hacer una aplicación en relación al método convencional $28 \%$ de las veces.

\section{AGRADECIMIENTO}

Al Ing. Agr. Ramón Fuentes por la elaboración inicial del anteproyecto de este estudio.

Al Dr. Bob OWeil por su ayuda en el análisis estadístico de este trabajo.

\section{BIBLIOGRAFÍA}

ANDREWS,K.L. 1984. El manejo integrado de plagas invertebradas en los cultivos hortícolas, agronómicos y frutales en la Escuela Agrícola Panamericana. El Zamorano, Honduras. 85 p.

; BARLETTA, H. 1985. Empoasca o lorito verde. Carta informativa para agricultores. Publicación MIPH-EAP No. 56. 1 p.

KING, A.B.S.; SAUNDERS, J.L. 1984. El manejo integrado de plagas invertebradas de cultivos anuales alimenticios en América Central. Overseas Development Administration. Londres. 182 p.

PORTILLO, H. 1988. Un método práctico de muestreo para Empoasca spp. en el cultivo del frijol. Tesis Ing. Agrónomo. Escuela Agrícola Panamericana. El Zamorano, Honduras. 27 p.

SCHOGNHOVEN, A.; CARDONA, C. 1985. Plagas que atacan el follaje. In Frijol: investigación y producción. CIAT, Cali, Colombia. p. 263-278.

SOBRADO, C.; ANDREWS, K.L, RUEDA, A.; PORTILLO, H. 1986. Un muestreador absoluto para Empoasca spp, In Reunión Anual del PCCMCA (32,1986, San Salvador, El Salvador). Memoria. San Salvador, El Salvador. 